\title{
CONTINUOUS RINGS WITH ACC ON ESSENTIALS ARE ARTINIAN
}

\author{
S. K. JAIN, S. R. LOPEZ-PERMOUTH, AND S. TARIQ RIZVI
}

(Communicated by Donald S. Passman)

ABSTRACT. It is proved that a left continuous ring with ascending chain condition on essential left ideals is left artinian.

\section{INTRODUCTION}

It is well known that for left or right self-injective rings diverse chain conditions on certain classes of left ideals are actually equivalent to the minimum condition on the lattice of all left ideals. Recently, Huynh-Dung and PageYousif proved independently that a left or right self-injective ring with ascending chain condition on essential left ideals is left artinian. In this paper we prove the preceding result by assuming that $R$ is left continuous instead of being left self-injective. Examples are given to show that one-sided continuity and one-sided chain conditions may not necessarily yield the continuity or chain conditions on the opposite sides.

\section{Preliminaries}

As defined by Utumi, a ring $R$ is called left continuous if (i) every left ideal of $R$ is essential in a direct summand of $R$ and (ii) every left ideal isomorphic to a direct summand of $R$ is itself a direct summand [6]. Continuous modules are defined analogously. Throughout, all rings have identity element and all modules are unital. $Z\left({ }_{R} R\right), J(R)$, and $\operatorname{Soc}\left({ }_{R} R\right)$ will denote, respectively, the left singular ideal, the Jacobson radical, and the left socle of a ring $R$.

We record below some well-known results often referred to in the proof of our theorem.

Lemma 1. If $R$ has acc on essential left ideals, then $Z\left({ }_{R} R\right)$ is nilpotent.

Proof. The proof is exactly the same as the one when $R$ has acc on left annihilators ([5, Lemma 3.39, p. 380]).

Received by the editors November 7, 1988.

1980 Mathematics Subject Classification (1985 Revision). Primary 16A34, 16A52; Secondary 16A36, 16A35.

Key words and phrases. Continuous rings, self-injective rings, chain conditions, essential left ideals. 
Lemma 2. Let $R$ be a left continuous ring. Then $J(R)=Z\left({ }_{R} R\right), R / J(R)$ is a von Neumann regular left continuous ring, and idempotents modulo $J(R)$ can be lifted.

Proof. See Utumi [6].

The following lemma has recently been proved independently by HuynhDung and Page-Yousif. For completeness we include here its proof by HuynhDung [2].

Lemma 3. For each left module $M, M$ has acc on essential submodules if and only if $M / \operatorname{Soc}(M)$ is left noetherian.

Proof. If $M$ has acc on essential submodules, each submodule $N$ of $M$ has the same property. Therefore, if $B \subset^{\prime} N \subset M, N / B$ is noetherian. In particular, it follows that every uniform submodule of $M$ is noetherian. Consider a complement $H$ of $\operatorname{Soc}(M)$. Then $\operatorname{Soc}(M) \oplus H \subset^{\prime} M$, and therefore $\frac{M}{\operatorname{Soc}(M) \oplus H}$ is noetherian. Since

$$
\frac{M}{\operatorname{Soc}(M) \oplus H} \simeq \frac{\frac{M}{\operatorname{Soc}(M)}}{\frac{\operatorname{Soc}(M) \oplus H}{\operatorname{Soc}(M)}} \quad \text { and } \quad \frac{\operatorname{Soc}(M) \oplus H}{\operatorname{Soc}(M)} \simeq H,
$$

it is now sufficient to show that $H$ is noetherian in order to obtain that $\frac{M}{\operatorname{Soc}(M)}$ is noetherian. Let $X=X_{1} \oplus X_{2} \oplus \cdots$ be a direct sum of nonzero submodules contained in $H$. Since for each $i, \operatorname{Soc}(M) \cap X_{i}=0$, it follows that each $X_{i}$ contains a proper essential submodule $Y_{i}$. Thus $Y=Y_{1} \oplus Y_{2} \oplus \cdots$ is an essential submodule of $X$, and therefore $\frac{X}{Y} \simeq \frac{X_{1}}{Y_{1}} \oplus \frac{X_{2}}{Y_{2}} \oplus \cdots$ is noetherian. Consequently, the sum $X=X_{1} \oplus X_{2} \oplus \cdots$ must be a finite sum, and therefore $H$ has finite Goldie dimension, say, $k$. Let $U=U_{1} \oplus \cdots \oplus U_{k}$ be a direct sum of uniform submodules which is essential in $H$. Since $H / U$ and $U$ are noetherian, $H$ is noetherian. This proves the claim. The converse is trivial.

Lemma 4. Let $M=\bigoplus \sum_{i=1}^{n} A_{i}$. Then $M$ is continuous if and only if each $A_{i}$ is continuous and $A_{j}$-injective for $j \neq i$.

Proof. This is a special case of [4, Theorem 13].

\section{THE RESULT}

Theorem. Let $R$ be a ring with ascending chain condition on essential left ideals.

(i) If $R$ is left or right continuous, then $R$ is semiperfect.

(ii) If $R$ is left continuous, then $R$ is left artinian.

(iii) If $R$ is right continuous, then $R$ need not be left artinian.

Proof. (i) By Lemma 2, $\bar{R}=R / J(R)$ is von Neumann regular and left (right) continuous. Because $\bar{R}$ is regular, the right socle of $\bar{R}=$ left socle of $\bar{R}=S$. Since $\bar{R}$ also satisfies the acc on essential left ideals, it follows from Lemma 
3 that $Q=\bar{R} / S$ is semisimple artinian. Let $M$ be a singular left (right) $\bar{R}$ module. Since $S M=0(M S=0), M$ is a $Q$-module and therefore $Q_{Q}\left(M_{Q}\right)$ is injective. This implies that $M$ is injective as an $\bar{R}$-module because $\bar{R}$ is regular. Next we show that every cyclic left (right) $\bar{R}$-module is continuous. For, let $\bar{I}$ be a left (right) ideal of $\bar{R}$. Then $\bar{I}$ is essential in $\bar{A}$, where $\bar{R}=\bar{A} \oplus \bar{B}$, since $\bar{R}$ is continuous. Thus, $\bar{A} / \bar{I}$ is singular and hence injective as an $\bar{R}$ module. Also, by Lemma $4, \bar{B}$ is $\bar{A}$-injective and so $\bar{B}$ is $\bar{A} / \bar{I}$-injective. Now from $\bar{R} / \bar{I} \simeq \bar{A} / \bar{I} \times \bar{B}$, it follows by invoking again Lemma 4 in the other direction that $\bar{R} / \bar{I}$ is continuous. Since a ring each of whose cyclic left (right) modules is continuous is always semiperfect $([3$, p . 201]), the regular ring $\bar{R}$ must be semisimple artinian. Hence by Lemma $2, R$ is semiperfect.

(ii) By Lemma 2 and Lemma $1, R$ is semiprimary. Write $R=\oplus \sum_{i=1}^{k} \operatorname{Re}_{i}$ as a direct sum of indecomposable left ideals. Since each $\operatorname{Re}_{i}$ is continuous, $\operatorname{Soc}\left(\operatorname{Re}_{i}\right)$ is simple or zero and so $\operatorname{Soc}\left({ }_{R} R\right)$ is finitely generated. Thus by Lemma $3, R$ is left noetherian and hence $R$ is left artinian because $R$ is semiprimary.

(iii) Let $R$ be a ring with only three right ideals $0, J(R)$, and $R$, which is not left artinian (see [1, Example 7.11'.1, p. 337]). $R$ is clearly right continuous. Also, $R$ has acc on essential left ideals since $J(R)=\operatorname{Soc}\left({ }_{R} R\right)$ (Lemma $3)$. Incidently, $R$ is not even left noetherian.

This completes the proof.

\section{REMARKS}

(i) It follows from our theorem and that of Utumi, ([6, Theorem 7.10]) that a left and right continuous ring with acc on essential left and essential right ideals is quasi-Frobenius.

(ii) Contrary to the fact that for a right self-injective ring $R$ the minimum condition on one side implies the minimum condition on the other side and the left self-injectivity of $R$, the example in part (iii) of the theorem shows that a right continuous right artinian ring need not be left artinian or left continuous.

(iii) A two-sided artinian ring with one-sided continuity is not necessarily continuous on the other side:

Let $R$ be a ring with only three left ideals $0, J(R)$, and $R$, which is a right artinian with composition length 3 ([1, Example 7.11'.2, p. 338]). $R$ is clearly left continuous. However, $R$ is not right continuous. For otherwise, by Remark (i), $R$ would be quasi-Frobenius, and hence uniserial with composition length 2 (on either side).

\section{REFERENCES}

1. C. Faith, Algebra: rings, modules, and categories I, Springer-Verlag, New York-Berlin, 1973.

2. D. van Huynh and N. V. Dung, A note on self-injective rings (preprint).

3. S. K. Jain and S. Mohamed, Rings whose cyclic modules are continuous, J. Indian Math. Soc. 42 (1978), 197-202. 
4. B. J. Müller and S. T. Rizvi, On injective and quasi-continuous modules, J. Pure Appl. Algebra 28 (1983), 197-210.

5. L. H. Rowen, Ring theory, Vol. 1, Academic Press, Boston, 1988.

6. Y. Utumi, On continuous rings and self-injective rings, Trans. Amer. Math. Soc. 118 (1965), 158-173.

Department of Mathematics, Ohio University, Athens, Ohio 45701

(S. Tariq Rizvi): Department of Mathematics, The Ohio State University, Lima, Ohio 45804 